\title{
Behavioural change effects on energy use in public housing: a case study
}

\author{
Andrea Sangalli ${ }^{1}$, Lorenzo Pagliano ${ }^{1}$, Francesco Causone ${ }^{1}$, Giuseppe Salvia ${ }^{2}$, Eugenio \\ Morello², Silvia Erba ${ }^{1}$ \\ ${ }^{1}$ end-use Efficiency Research Group, Department of Energy, Politecnico di Milano, \\ via Lambruschini 4, 20156 Milano, Italy \\ ${ }^{2}$ Department of Architecture and Urban Studies, Politecnico di Milano, \\ Piazza Leonardo da Vinci 32, 20133 Milano, Italy
}

\begin{abstract}
Post-occupancy evaluations of building energy performances after retrofit often show values of energy use higher than calculated during the design phase. This performance gap is due to different factors, among which the user behaviour plays a crucial role. In the context of public housing, in particular, the techno-centric approach usually adopted during all the work phases tends to omit the involvement and information of the inhabitants, leading to ways of living not consistent with optimal uses of the building services and components. To tackle this limit, EnerPOP, a project funded by Politecnico di Milano, is developing a methodology for reconciling technical and social aspects in the refurbishment of public housing assets. A case study is adopted to experiment and optimise the methodology, engaging tenants of a public residential building in Milan, deeply renovated in 2014 and inhabited by 500+ people originating from over 30 different countries. The paper reports interim findings, after one year of activities, both in terms of knowledge of the building and its inhabitants and early results in terms of energy saving and social cohesion.
\end{abstract}

Keywords: Energy Retrofit, Rebound Effect, User Behaviour, Performance Gap.

\section{Introduction}

Starting from 2016, the Municipality of Milan invested 103.5 million euros, distributed over 4 years, for the refurbishment of public residential buildings (project "Zero case vuote” - “Zero uninhabited houses”) [1]. Energy efficiency measures are a relevant part of this plan; however, the planning, implementation and management of energy efficiency interventions are quite often carried out with a techno-centric approach. This may lead to unintended consequences, such as lower than expected energy savings actually achieved by projects. This "rebound effect" may be better understood if occupants' routines and ways of living are considered [2], since these may not coincide with 
the expected ones at the design stage ${ }^{1}$. The rebound effect may be framed in very different ways depending on various factors, including the social context, the degree of technological innovation of the solutions adopted in the refurbishment, the configuration of properties (public or private, rental or home ownership) [3,4].

EnerPOP, a project funded by pre-tax donations for Politecnico di Milano, aims to analyse the technical and social aspects in a public residential building retrofit case study in Milan, in order to identify the causes of the gap between the expected (calculated) energy savings and the actual (measured) ones, and to propose options and perform actions to reduce it. In two previous studies by the same authors [5,6], the starting situation has been analysed from a technical and social perspective, respectively. This work is intended as a follow-up, with the aim (i) to upgrade the above-mentioned analysis, for a better understanding of the occupants' behaviours demanding energy, and (ii) to show the first results in terms of energy savings and social interactions, after one year from the beginning of the project.

\section{Nomenclature}

HDD Heating degree days

\section{Description of the case study}

The case study is a large public residential building, owned by the Municipality of Milan, located in a context of marked urban and social periphery. It is a five stories building, with 154 apartments, about half of which are small two-rooms apartments of about $40 \mathrm{~m}^{2}$, and the rest four-rooms apartments with a surface ranging from $65 \mathrm{~m}^{2}$ to $85 \mathrm{~m}^{2}$, for a total of about $11,000 \mathrm{~m}^{2}$ of gross floor area [5].

Since 2013, the building has undergone a major energy refurbishment. The original building was characterized by poor energy performance of the envelope and by obsolete mechanical systems, with resulting thermal discomfort (low air temperatures and radiant temperatures in winter) and low air quality (diffuse presence of mould). The renovation work focused first on improving the quality of the envelope and on the renewal

${ }^{1}$ In this paper, the term "behaviours" is used to refer to occupants' ways of living in the house, including those using energy for heating and achieving thermal comfort in general. Nevertheless the authors are aware of the ongoing theoretical debate in energy use and comfort studies between approaches informed by psychological approaches related to behavioral change on the one hand and on the other the sociology informed one addressing social practices and their configurations (cfr. Shove, E. 2010. Beyond the ABC: Climate Change Policy and Theories of Social Change. Environment and Planning A: Economy and Space, 42(6), 1273-1285. https://doi.org/10.1068/a42282). Although relevant, the elements of the debate fall outside the scope of this paper, which aims mainly at sharing preliminary insights on energy monitoring analysis. In this publication the term "behaviours" is preferred for consistency with the contribution in the specific field of the conference and reduce risks of comprehensibility. Forthcoming publications from the EnerPOP project intend to unpack how framing the investigation through Social Practice Theory may provide relevant insights especially on the ways tenants arrange their routines and the consequences on energy demand. 
of the heating system, with the centralization of hot water production as well as heating, followed by the connection to the district heating network. In each apartment, a programmable thermostat and thermostatic valves have been installed on the radiators, for control purposes only (billing is not based on individually metered consumption). A centralized mechanical ventilation system was also installed, which operates in extraction only, with extraction vents placed in the bathrooms and fresh air entering the apartments through intake vents placed on the façade. The system works with constant flow and at low speed. In 2014, at the end of the retrofit works, the building was certified "class B" according to the Italian energy performance classification, with a calculated total primary energy use for heating of $34 \mathrm{kWh} /\left(\mathrm{m}^{2} \mathrm{yr}\right)$.

Towards the end of 2014, the apartments were progressively assigned to the tenants, which were not the same ones previously occupying the building. At present, the community that resides in the building consists of about 500 people, mostly elderly Italians and families of first-generation immigrants. About $30 \%$ of the inhabitants are under the age of 15 - a particularly young community compared to the average of the public housing in Milan. About the $60 \%$ of the tenants belong to families whose head originates from a foreign country rather than Italy, including more than 30 different nationalities. In all families, at least the head of the family is able to speak Italian.

\section{$3 \quad$ Methodology}

In order to gain knowledge about the post-retrofit situation, data on energy use for heating and electrical energy use have been gathered for all the apartments since 2014; whereas data on indoor thermal comfort and indoor air quality have been gathered for a selected subset of apartments since 2016. The thermal energy meters, installed in each apartment on the heating manifold, measure the thermal energy required to reach and maintain the value of air temperature set on the programmable thermostat. The measured energy does not comprise the distribution efficiency of the part of the system placed inside the apartment (between the collector and the radiators) and the emission efficiency of the radiators. The measured value corresponds approximatively to the "energy need for space heating" according to the terminology of related standard ISO 52016-1: 2017. Some data are missing over the years in some apartments (due to end of battery charge and sudden sensor failures). Unfortunately, these issues were not solved promptly due to a lack in the maintenance chain between the building owner and the maintainer. Since 2015, 95 over 154 apartments were continuously monitored without failures. Only these apartments have been considered in the following analysis, in order not to alter the characteristics of the dataset (e.g. the influence of the position of the apartments, households involved, ...). In the considered apartments, residents have not changed substantially over the years, therefore the results should be comparable.

Table 1 summarizes the energy use for heating of all the apartments where the energy meters worked continuously since 2015, showing the average for the heating seasons 2015-16, 2016-17 and 2017-18, compared to the last one, 2017-18. The first available heating season (2014-15), has not been considered, because the apartments were progressively assigned since October 2014 and the related lower energy uses depended on 
non-occupancy. Data are presented as (i) normalized per surface of the available apartments, and (ii) furtherly normalized with respect to the heating degree days (HDD) of the respective seasons ${ }^{2}$. The comparison with the last heating season (2018-19), after the first year of the EnerPOP activities, shows a reduction of $11 \%$ in the energy use for heating; the percentage is even greater considering, in the comparison, the last season before EnerPOP (2017-18), instead of the average 2015-18; in this case, the normalized variation is $-16 \%$.

Table 1. Average values of the energy use for heating (absolute and normalized to HDD) for the 95 apartments

\begin{tabular}{lccc}
\hline & $\begin{array}{c}\text { Average } \\
\mathbf{2 0 1 5 - 1 8}\end{array}$ & $\begin{array}{c}\mathbf{2 0 1 8 - 1 9} \\
\text { (EnerPOP) }\end{array}$ & Variation \\
\hline $\mathbf{k W h} /\left(\mathbf{m}^{2} \mathbf{y r}\right)$ & 81 & 67 & $-17 \%$ \\
\hline $\mathbf{k W h} /\left(\mathbf{m}^{2} \mathbf{y r}\right.$ HDD) & 0.040 & 0.035 & $-11 \%$ \\
\hline
\end{tabular}

Regarding environmental monitoring, since December 2016, temperature, humidity, and $\mathrm{CO}_{2}$ sensors have been installed in 17 apartments. They are placed in a barycentric position of the apartment, far from heat sources or thermal dispersions, at a height of $150 \mathrm{~cm}$ from the floor, on the side of the programmable thermostat; it can, therefore, be assumed that the monitored air temperature corresponds to that used by the controls of the heating system. In the monitored two-level apartments, an additional sensor was installed on the upper floor. The environmental data are recorded hourly. At the moment, the visualization of the data is not available for the tenants.

Fig. 1 shows the normalized energy use and the statistical distributions of the temperature values, for the apartments in which both parameters are measured. The values are sorted by increasing energy use, for the heating season 2018-19, together with the corresponding temperatures, whose statistical distributions are represented by a boxand-whisker graph. In this graph, the quartiles are indicated by blue bars (first quartile, q1, second quartile, or median, q2, and third quartile, q3) and the maximum and minimum values, included respectively in the interval [q3 + 1.5* $(\mathrm{q} 3-\mathrm{q} 1)]$ and [q3 -1.5 * (q3 - q1)], are represented as whiskers above and below the quartiles. The values are compared with the ones of the previous heating season 2017-18, represented with dotted lines, both for the energy use and for the median temperatures.

The variation in energy use for heating over the last two seasons appears to be nonhomogeneous: 5 out of 14 apartments show a significant reduction, 5 are quite similar, while 4 increased their values. A certain correlation between the variation of temperatures and energy use is shown in some apartments $(48,112,47,144,73,42,100,154)$, while the other ones present counter-intuitive trends.

\footnotetext{
2 The HDD have been calculated on an hourly basis, according to ISO 15927-6: 2007. "Heating season” here means the period from October 15 to April 15 , according to the Italian laws $n^{\circ} 10$ of 9/1/1991 and DPR 412 of 26/8/1993 and subsequent amendments, as a period of operation of heating systems in buildings in the municipalities belonging to the climate zone "E", like Milan.
} 


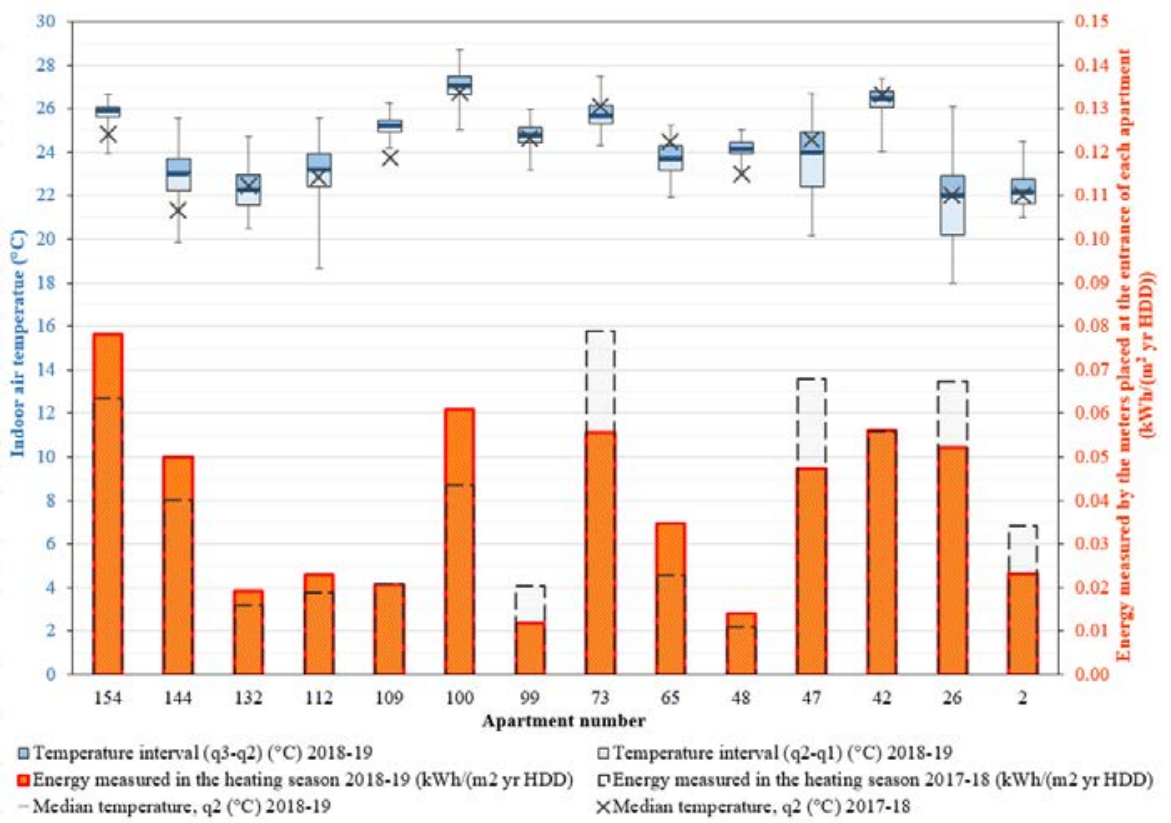

Fig. 1. Heating season 2017-18 and 2018-19: energy measured by the meters placed at the entrance of each apartment and temperatures in the corresponding apartments (the number of which is invented by the authors and do not correspond to actual ones to preserve the tenants' privacy).

An analysis has been carried out also on the energy use for electricity, comparing, for some apartments, the consumption of two months of winter with those of two months of summer of the same year. Households with higher energy use for electricity in summer probably use active cooling with individual devices, since no active cooling was provided by the municipality. This possibility was suggested also during interviews with tenants. In order to have empirical confirmations, an additional comparison was done with indoor temperatures recorded in June-July, as shown in Fig. 2. Except for apartment 65 , no particular correlations seem to be drawn at the moment between the two parameters. The reasons may be related to the typical limited and discontinued use of cooling: if only one room is cooled, the temperature recorded by the monitoring system may be not representative, and if cooling is activated only in some hours, those few occurrences are outliers which have not been represented in the statistical evaluation of the temperatures over the whole hours of June-July. Additional work is therefore needed with this respect, either installing additional sensors in some apartments, to have a more comprehensive representation of the indoor temperatures over the whole apartment, or trying to draw occupation hours, deriving them from the analysis of indoor $\mathrm{CO}_{2}$ levels over time or from electrical load curves. 


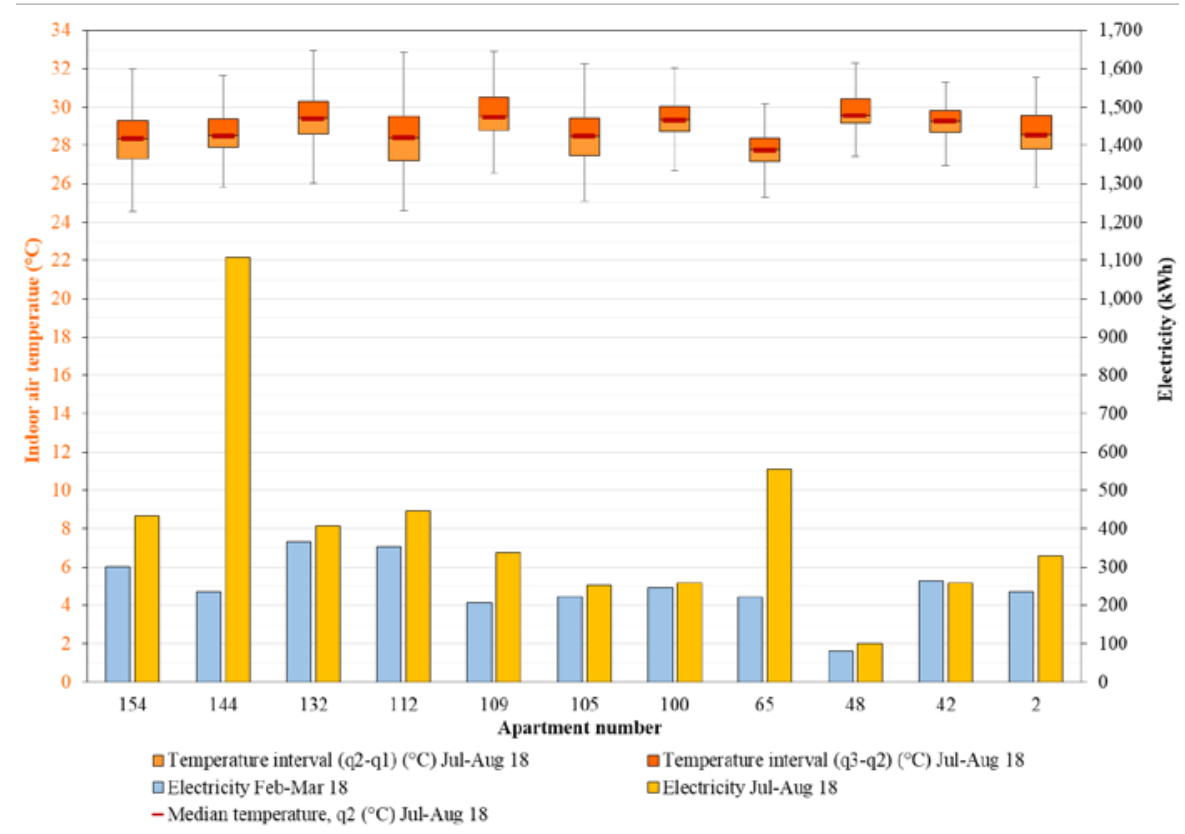

Fig. 2. Energy use for electricity in two months (in winter and summer, respectively) in some apartments, compared to the indoor temperatures.

A long-term comfort analysis was also performed based on the air temperature values measured in the apartments during the heating season 2018-19, in comparison to those already performed for 2016-17 and 2017-18 [5]. Fig. 3 presents the comparison between the analysis for the heating season 2017-18 and 2018-19. The graphs show that, although conditions in most of the apartments fall most of the time into category II as required by the standard ${ }^{3}$, there are also several situations characterized by overheating with respect to category II. These occurrences appear to decrease in 2018-19, in some cases, with an increase of undercooling two apartments.

Another long-term analysis was carried out, this time related to indoor air quality, evaluated by measuring the $\mathrm{CO}_{2}$ concentrations in some apartments and comparing them with the category limits set by the standard EN 15251. The hours when the $\mathrm{CO}_{2}$ concentrations fall in the various categories were counted, comparing them to the total number of hours in the considered period, thus obtaining the percentages shown in Fig. 4.

\footnotetext{
${ }^{3}$ EN 15251 Annex G: "The different parameters for the indoor environment of the building meet the criteria of a specified category when: the parameter in the rooms representing $95 \%$ of the occupied space is not more than for example $3 \%$ (or $5 \%$ ) of occupied hours a day, a week, a month and a year outside the limits of the specified category [...]”.
} 

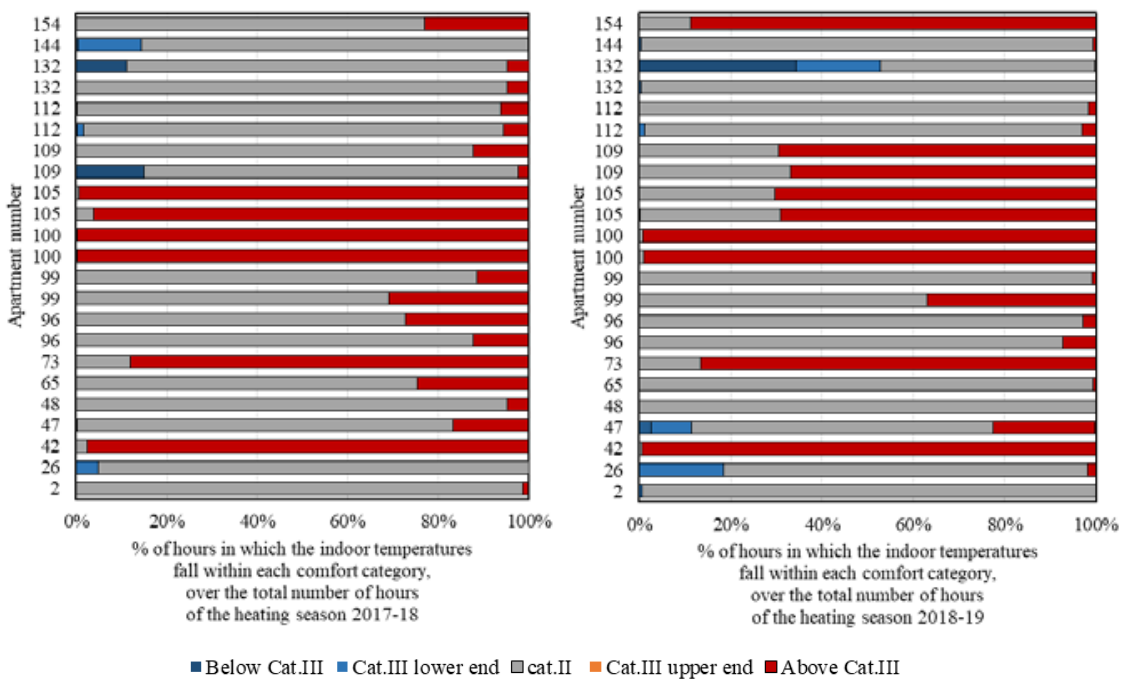

Fig. 3. Percentage distribution of hours in the various comfort categories during the heating period 2017-18 and 2018-19 for the apartments where the indoor environmental sensors were installed. Situations where the temperature is comprised between the upper limit of the cat. N-1 and the upper limit of cat. $\mathrm{N}$ are labelled by "upper end" and situations where the temperature is comprised between the lower limit of the cat. $\mathrm{N}$ and the lower limit of cat. $\mathrm{N}-1$ are labelled by "lower end". E.g. "Cat.II lower end" means that the temperature is between the lower limit of cat. II and the lower limit of cat. I.

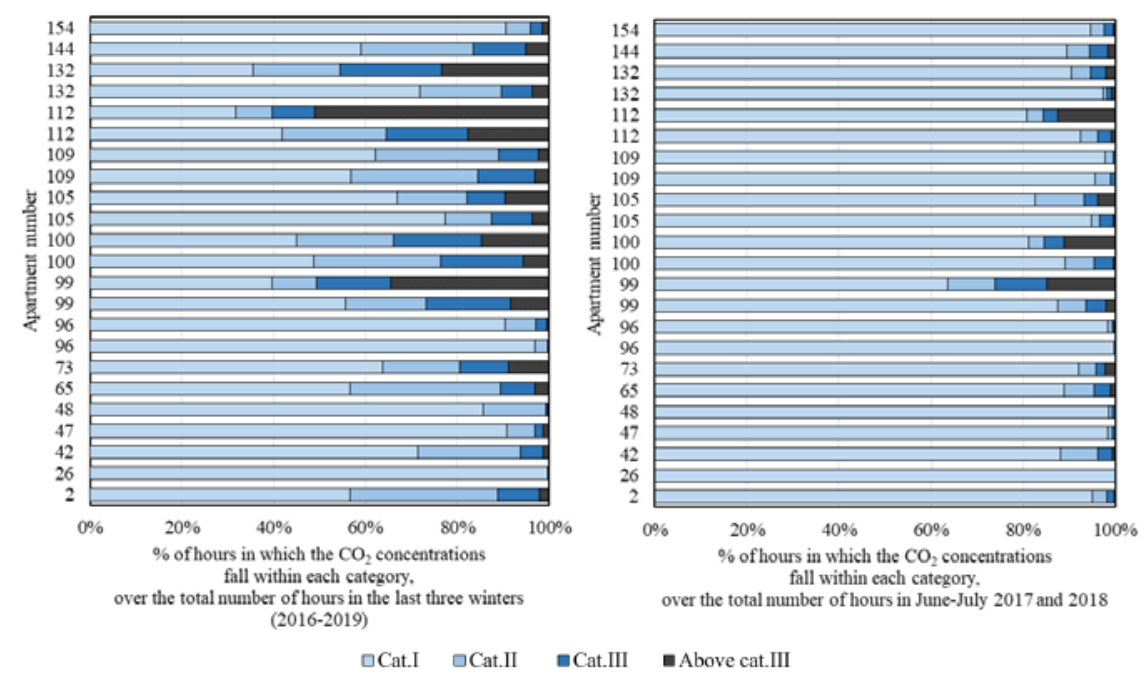

Fig. 4. Percentage distribution of hours in the various categories of $\mathrm{CO}_{2}$ concentrations, for the apartments where the indoor environmental sensors were installed. 
In parallel to physical measurements, information has been collected about the tenants' habits, by conducting semi-structured interviews to a sample of tenants, as well as conversations at different levels of formalization (e.g. scheduled meetings, unscheduled visits) and diverse participants' observations. The choice of the sample of tenants was initially aiming to an equitable representation for the placement of the apartment (floor, exposure), size of the family (single, couple, family with children), nationality. The selected people were asked to participate in an interview lasting about 60 minutes in their home, on a voluntary basis (without reimbursement) and according to their time preferences (date and hour). The analysis of the interviews shows a wide variability of social behaviours and descriptors of thermal comfort, as expected. The difficulty in understanding how the building systems work and how to maintain the ideal temperature at home is common, and it influences the interaction with programmable thermostat, thermostatic valves, windows and accessory devices to achieve the ideal temperature, both in summer and in winter.

\section{$4 \quad$ Discussion of the results}

As shown in Table 1, in the last winter 2018-19, the energy use for heating presented an overall reduction of $11 \%$ with respect to the average of the previous winters. Since the data are normalized to the HDD, they take into account the different severity of the outdoor conditions over the years. Therefore, other explanations can be sought for this variation, assuming that random variability alone is not enough. Other variations did not take place, such as variation in the social composition, in the billing or in the characteristics of the envelope and of the heating system. Due to this, the activities of involvement carried out in the first year of the EnerPOP project, may be assumed responsible of a consistent part of the energy savings, because they enabled a rearrangement of tenants' behaviours, which may have generated also a positive, environmentally sustainable impact. The main activities explicitly addressing thermal comfort in winter and energy savings consisted of a campaign informing about the prescription by law concerning the temperature to be set in the buildings, i.e. $20^{\circ} \mathrm{C}$, and the maximum number of hours in which the heating system can be working, i.e. $14 \mathrm{~h}$, information for a better use of the programmable thermostat; not keeping windows open to lower the temperature, but doing it with a proper regulation of the programmable thermostat and of the thermostatic valves on the radiators, other than specific interaction by the researcher with some of the tenants, to help them to regulate the programmable thermostat. The campaign consisted of multiple channels including:

1. tutoring, i.e. researchers supporting tenants onsite;

2. the projection of a video on optimal behaviours (e.g. setting lower temperature, keeping windows closed...) co-designed and partly co-created with the tenants, played continuously in a loop, 24 hours a day for several days, in the common spaces at the entrance of the building;

3. a video including instructions on how to set the programmable thermostat, step by step;

4. a leaflet distributed to all the tenants' mailboxes; 
5. a notice placed on the communal areas with the link to the online page where the video on the programmable thermostat could be visualized.

Assuming that these actions were effective in a more energy-efficient reconfiguration of behaviours for indoor thermal comfort of the tenants, it is possible to inquire more in-depth about the reasons behind this effectiveness.

First, the multi-channel campaign may have met the more or less evident demand of information and support with regard to the ways of configuring the available devices, mainly the programmable thermostat, which was reportedly assessed as obscure to interpret. In fact, some tenants welcomed the researchers in their houses to solve problems with their radiators, which occasionally were due to the programmable thermostats.

Second, the campaign may have provided information on the legal terms for the heating setting, which were not widely known (e.g. $20^{\circ} \mathrm{C}$ max at home, for 14 hours maximum). In a couple of interviews held after the launch of the campaign highlighted how children may represent an advantageous means for this, either as they stopped to watch the video together with their parents or because they reported the contents back to the parents afterwards.

Third, the campaign may have inadvertently created the impression of local monitoring which may have discouraged some tenants to abuse of the heating, thus facing risks of receiving a fine. Such case may not be ideal to enable a longer-term transition towards more energy efficient thermal behaviours; nevertheless, this would suggest that holding initiatives on the topic generates an impact.

Despite the limited number of tenants reached directly by the researchers, possibly due to mistrust, the impact seems to have been larger than expected. This may be due to communications between the tenants by word-of-mouth.

\section{Conclusions}

After the first year of field research, it clearly emerges how the actual energy use of a building and the connected energy costs (often paid by the Municipality, in public housing contexts) may present significant deviations in comparison to the values estimated during the design phase, even in case of careful design and energy modelling, according to standards and protocols and careful execution of the retrofit work. The actual behaviour carried out in the apartments may differ considerably with respect to the modelled ones, most notably about the timespan windows are kept open and shutters rolled up, with related heat dispersion and wasted energy.

The presented analyses show a reduction of $11 \%$ in the energy use for heating, compared to the average of the previous three heating seasons, normalized to HDD. In the absence of other explanations, it can be assumed that this result is related to the EnerPOP activities, which started a process of rearrangements of tenants' behaviours towards more environmentally sustainable ways of living.

The current and future activities of the project aim at a more detailed definition of the behaviours conducted in the homes by the tenants, increasing the representativeness of 
the descriptions provided, and at an identification of patterns of habits, needs, perceptions of comfort, cultural factors which might provide explanatory variables of the variations in energy use. Finally, it is foreseen to develop further actions to support tenants to identify and modify inefficient behaviours, with a process focused on participation and empowerment.

\section{Acknowledgments}

The research has been partially funded by the Polisocial Award 2017 of Politecnico di Milano under the EnerPOP project and by the European Union's Seventh Framework Program for research, technological development, and demonstration under grant agreement No ENER/FP7EN/314632/EU-GUGLE. We are thankful to the partners of the projects and in particular Arch. Manzoni and Arch. Bardeschi of Milan Municipality for their useful collaboration.

\section{References}

1. Retrieved from https://milano.corriere.it/notizie/cronaca/18_gennaio_24/milano-case-popolari-alloggi-sfitti-il-recupero-stanziati-1035-milioni-02fc3aa2-00d0-11e8-b515cd75c32c6722.shtml.

2. Gram-Hanssen K. 2014. Retrofitting owner-occupied housing: remember the people, Building Research \& Information, 42:4, 393-397, DOI: 10.1080/09613218.2014.911572.

3. Atkinson J., Littlewood J., Karani G., Geens A. 2016. Relieving fuel poverty in Wales with external wall insulation. Proceedings of the Institution of Civil Engineers - Engineering Sustainability, 170(2), 93-101.

4. Sousa Monteiro C., Causone F., Cunha S., Pina A., Erba S. 2017. Addressing the challenges of public housing retrofits. Energy Procedia, 134, 442-451.

5. Sangalli, A., Pagliano, L., Causone, F., Salvia, G., Morello, E. (2019) Energy efficiency and occupants' behavior: analysis of a public housing case study. In proceeding of AICARR 51st international conference, Venice 20-22 February.

6. Salvia G, Rotondo F, Morello E, Sangalli A, Pagliano L, Causone F. (2019) Sustainability designed with(out) people? Understanding for what energy is (over-)used by tenants in an energy efficient public housing in Milan. In proceedings of LENS international conference, Milan 3 April. 